\title{
The Neural Correlates of Positive Versus Negative Thought-action Fusion in Healthy Young Adults
}

\author{
Sang Won Lee ${ }^{1,2, *}$, Hyunsil $\mathrm{Cha}^{3, *}$, Tae Yang Jang ${ }^{2,4}$, Eunji $\mathrm{Kim}^{3}$, Huijin Song ${ }^{5}$, Yongmin Chang ${ }^{6,7}$, \\ Seung Jae Lee $^{2,4}$ \\ ${ }^{1}$ Department of Psychiatry, Kyungpook National University Chilgok Hospital, Departments of ${ }^{2}$ Psychiatry and ${ }^{3}$ Medical \& Biological Engineering, \\ School of Medicine, Kyungpook National University, ${ }^{4}$ Department of Psychiatry, Kyungpook National University Hospital, Daegu, ${ }^{5}$ Biomedical \\ Research Institute, Seoul National University Hospital, Seoul, ${ }^{6}$ Department of Molecular Medicine, School of Medicine, Kyungpook National \\ University, ${ }^{7}$ Department of Radiology, Kyungpook National University Hospital, Daegu, Korea
}

Objective: Thought-action fusion (TAF), one of the most-studied dysfunctional beliefs in obsessive-compulsive disorder, represents an individual's belief that his/her thoughts directly influence events. TAF belief types are divided into personal thoughts relating to positive (positive TAF) and negative outcomes (negative TAF). However, the neural mechanisms underlying both aspects of the TAF response remain elusive.

Methods: This functional magnetic resonance imaging study aimed to investigate the neural circuits related to positive and negative TAF and their relationships with psychological measures. Thirty-one healthy male volunteers participated in a modified TAF task wherein they were asked to read the name of a close person embedded in positive statements (PS) or negative statements (NS).

Results: Conjunction analysis revealed activation of the fusiform and lingual gyri, midcingulate and superior medial frontal gyri, inferior orbitofrontal gyrus, and temporoparietal junction. The NS > PS comparison showed additional activation in the precuneus and medial prefrontal cortex, superior frontal gyrus, insula, globus pallidus, thalamus, and midbrain. Precuneus activity was associated with the TAF score among these areas. Moreover, activity in the inferior orbitofrontal gyrus, insula, superior, middle and medial frontal gyri, globus pallidus, inferior parietal lobule, and precuneus was associated with dimensional obsessive-compulsive scores. In contrast, the PS > NS comparison revealed no significant activation.

Conclusion: These results suggest that negative TAF, relative to positive TAF, recruits additional regions for self-referential processing, salience, and habitual responding, which may contribute to the activation of the belief that a negative thought increases the probability of that negative outcome.

KEY WORDS: Thought-action fusion; Cognitive theory; Obsessive-compulsive disorder; Cortico-striato-thalamo-cortical circuit; Affective network; Salience network.

Received: August 3, 2020 / Revised: November 3, 2020

Accepted: December 11, 2020

Address for correspondence: Seung Jae Lee

Department of Psychiatry, School of Medicine, Kyungpook

National University, 680 Gukchaebosang-ro, Jung-gu, Daegu

41944, Korea

E-mail: jayleemd@knu.ac.kr

ORCID: https://orcid.org/0000-0003-3648-9824

Yongmin Chang

Department of Molecular Medicine, School of Medicine,

Kyungpook National University, 680 Gukchaebosang-ro, Jung-gu,

Daegu 41944, Korea

E-mail: ychang@knu.ac.kr

ORCID: https://orcid.org/0000-0002-0585-8714

*These authors contributed equally to this study.

\section{INTRODUCTION}

A Korean proverb says "words can be a seed." It is translated in English as "disaster comes from the mouth." In this context, a seed can be understood as the cause of something that happens in the future. This proverb warns us not to speak of or even think about bad expectations based on the belief that things people say usually have a way of coming true. This phenomenon, referred to as thought-action fusion (TAF), has drawn the attention of cognitive theorists studying the underlying maladaptive assumptions in patients with obsessive-compulsive disorder (OCD). More recently, acceptance and commitment therapy has used

@) This is an Open-Access article distributed under the terms of the Creative Commons Attribution Non-Commercial License (http://creativecommons.org/licenses/by-nc/4.0) which permits unrestricted non-commercial use, distribution, and reproduction in any medium, provided the original work is properly cited. 
the terms believability in particular and cognitive fusion in general for this tendency [1-3]. Cognitive fusion is a process that involves attaching a thought to an experience in which literal verbal rules tend to dominate other sources of information and make people avoid actual experiences or the so-called experiential avoidance [4].

TAF is the belief that thought and outcome or action are linked [5]. The original TAF phenomenon was categorized into two types - moral and likelihood - with the latter further divided into likelihood-self and likelihoodother. Moral TAF involves the judgment that having an undesirable thought is morally equivalent to engaging in the action, while likelihood TAF reflects the belief that a thought increases the probability of that event. From a different angle, likelihood TAF can engage in a continuum from the positive (e.g., "If I think of my mother winning the lottery, this increases the probability of her winning") to the negative outcomes or events (e.g., "If I think of my husband being involved in a motor vehicle accident, this increases his risk of a car accident”). These two biases, labeled positive and negative TAF, do exist as independent constructs [6].

Negative TAF has been extensively examined in studies of OCD and various mood disorders [7-9]. Dysfunctional beliefs such as negative TAF have been proposed to play an important role in the development and maintenance of the disorder in the cognitive appraisal models of OCD $[10,11]$. TAF increases the perceived significance of a thought or obsession, which in turn leads to maladaptive safety behaviors, such as excessive preoccupation with thoughts, avoidance, or neutralization, in an attempt to avert disastrous consequences $[5,12]$. Further, exaggerated TAF responses occur not only in patients with OCD but also in those with generalized anxiety disorder [13], schizotypy [14], and major depressive disorder $[15,16]$.

Positive TAF in contrast with negative TAF has been much less investigated. Positive items were originally included in the TAF likelihood subscales but were eventually eliminated from the revised version of the TAF scale because they are least relevant to OCD [5]. Some investigators have proposed that positive likelihood TAF can operate in patients with OCD to prevent harm in harm avoidance, mental neutralizing, or the use of covert compulsions $[7,17]$. In addition, the relationship between positive TAF and impaired control of mental activities suggests that positive TAF may also be related to obsessive- compulsive (OC) tendencies [18]. However, avoiding harm is not the same as creating a positive outcome. In another context, the positive TAF bias is hypothesized to increase personal desire to obtain positive outcomes believed to result from thoughts. This suggests that positive TAF may be related to various risk-taking behaviors, such as gambling [6].

The neural mechanisms underlying the TAF response remain elusive despite its relevance in psychopathology. However, a few studies exist investigating the neural correlates of the negative likelihood TAF. One previous electroencephalogram (EEG) study showed that precuneus activity was positively correlated with the likelihood TAF score [19]. In addition, a recent functional magnetic resonance imaging (fMRI) study demonstrated increased activity in the lingual gyrus, caudate nucleus, precuneus, and several areas of the frontal cortex during a TAF induction task [20]. However, it is believed that no previous study has used fMRI to elucidate the neural circuits related to the positive TAF response. Moreover, no direct comparison exists between the two aspects of TAF using fMRI. A previous fMRI study conducted by the authors of the current study compared only the responses to a close and neutral person in the same context of negative TAF statements. Hemodynamic activity in the negative TAF would more clearly contrast with that in the positive TAF in a comparative assessment of both aspects.

This fMRI study aimed to investigate the neural circuits related to the positive and negative TAF using a modified TAF task wherein individuals were asked to read the name of a close person embedded in positive statements (PS) or negative statements (NS) such as "I hope $\cdots$ wins the lottery" or "I hope $\cdots$ is in a car accident" [21]. This study tried to clarify the common and differential patterns of brain activation for each TAF. NS tend to heighten TAF more than PS although a paucity of literature is noticed on positive TAF based on a previous study [17]. Thus, it is hypothesized that regions more highly activated in negative than in PS would be core areas that enhance TAF tendency, and the activity would correlate with conventional TAF scores and associated psychopathologies, including OC symptoms. 


\section{METHODS}

\section{Participants}

Thirty-one healthy male undergraduates recruited through an online bulletin board at Kyungpook National University participated in this study. Two experienced psychiatrists (SWL and SJL) conducted psychiatric interviews on all participants to exclude existing psychiatric pathology, psychotic symptoms, intellectual disability, neurological disease, and history of head injury or medical illness with documented cognitive sequelae. The mean \pm standard deviation age of the subjects, all right-handed, was $22.9 \pm$ 1.9 years. All participants provided written informed consent according to the procedures approved by the Institutional Review Board of Kyungpook National University Hospital (approval no. 2018-04-029).

\section{Psychological Measures}

\section{Thought-Action Fusion Scale (TAFS)}

The TAFS is a 19-item self-reported measure that assesses the tendency to fuse thoughts and actions. Each item is rated on a 5-point scale ranging from 0 (disagree strongly) to 4 (agree strongly). Twelve, four, and three items assess moral TAF, likelihood TAF-other, and likelihood TAF-self, respectively [5]. The validated Korean version of the TAFS was used [22].

\section{Obsessive-Compulsive Inventory-Revised (OCl-R)}

The OCl-R is an 18-item self-reported questionnaire that assesses the degree of distress caused by OCD symptoms. Each of the items is rated from 0 (not at all) to 4 (extreme), and three items constitute a subtype of OCD symptoms [23]. A validated Korean version of the OCI-R was used [24].

\section{Dimensional Obsessive-Compulsive Scale (DOCS)}

The DOCS is a 20-item self-reported measure [25] that assesses the severity of the four most consistently replicated OC symptom dimensions of (a) contamination/ washing, (b) harm obsessions/checking compulsions, (c) unacceptable thoughts, and (d) symmetry/ordering. Each item is rated on a $0-4$ scale, yielding a total score that ranges from 0 to 80 as well as individual subscale scores ranging from 0 to 20. A validated Korean version of the DOCS was used [26].

\section{Beck Depression Inventory (BDI)}

The BDI $[27,28]$ is a self-report measure containing 21 items on a 4-point scale from 0 (symptom absent) to 3 (severe symptoms). Scoring is achieved by adding the highest ratings for all 21 items. A validated Korean version of the BDI was used [29].

\section{TAF Induction fMRI Paradigm}

The participants were asked to name two close living persons, one male and one female, before the MR scan. These names were then used in the TAF paradigm. The names of one male and one female were presented in each condition to balance for sex. The closeness of the two close persons was $8.7 \pm 2.0$ on a $1-10$ scale of closeness per person. Eight PS and eight NS were used. An example of the former is "I hope that the person (his or her name, previously typed) will win a lottery." On the other hand, an example of the latter is "I hope that the person will soon be in a car accident."

The TAF FMRI paradigm used in this included two conditions, PS and NS, with eight statements for each condition. Each trial for each statement comprised four phases (e.g., name, sentence, evaluation, and a resting phase). First, the participants were asked to think about the close person while watching the name of that person being displayed on the screen for 4 seconds (name phase). Second, the participants were instructed to silently read the subsequent PS or NS for 10 seconds (sentence phase). Third, the participants then rated how badly or gladly they felt about the statement on a Likert scale from 1 (very little) to 4 (very much) using the magnetic resonance convertible response device with four buttons for another 4 seconds (evaluation phase). Finally, the participants were asked to look at a cross at the center of the screen for 10 seconds (resting phase). The order of the statements, NS and PS, was fixed, while two close persons' names, one for each sex, were intermixed in a pseudorandomized order. The TAF paradigm lasted for 7 minutes, 28 seconds (28 seconds for each trial $\times$ eight statements for each condition $\times$ two conditions). This TAF paradigm was adapted from a previous report [21] and modified for fMRI experiments [20].

\section{Image Acquisition and Analysis}

The functional images were obtained on a 3.0T MRI scanner (Discovery MR750w; GE Healthcare, Milwaukee, 
WI, USA) using a 24-channel head coil. A T2*-weighted gradient echo planar imaging pulse sequence was used for functional imaging (repetition time $[T R]=2,000 \mathrm{~ms}$, echo time $[\mathrm{TE}]=30 \mathrm{~ms}$, flip angle $[\mathrm{FA}]=90$, field of view [FOV] $=23 \mathrm{~cm}$, acquisition matrix $=64 \times 64$, and no gap). In addition, a three dimensional T1-weighted fast spoiled gradient echo was used for the acquisition of structural images $(\mathrm{TR}=8.5 \mathrm{~ms}, \mathrm{TE}=3.2 \mathrm{~ms}, \mathrm{FA}=13, \mathrm{FOV}=25.6$ $\mathrm{cm}$, and acquisition matrix $=256 \times 256)$. Statistical parametric mapping program (SPM12; Wellcome Trust Centre for Neuroimaging, University College London, London, UK; http://www.fil.ion.ucl.ac.uk/spm) and MATLAB (The MathWorks, Inc., Natick, MA, USA) were used for fMRI data image processing and statistical analyses. The functional images were preprocessed with slice timing, realignment, normalization into the standardized Montreal Neurological Institute ( $\mathrm{MNI}$ ) brain space, and spatial smoothing with a Gaussian kernel. Maps of brain activation for each condition were determined by a one-sample $t$ test in the within-group analysis. The threshold of the SPM(t)s one-sample $t$ test at family-wise error (FWE)-corrected voxel-wise was $p<0.05$. Differences in brain activation between the PS and NS conditions were analyzed using the paired comparison analysis while the conjunction analysis was performed for the regions that were commonly activated. The threshold of the above analyses at false discovery rate (FDR)-corrected voxel-wise was $p<$ 0.05 . A minimum cluster size of 10 was selected to report the results.

\section{Statistical Analysis}

The contrast name plus sentence phase was focused on, among the four phases of the TAF paradigm of this study, as the main outcome in each of the PS and NS conditions to explore whether similarities and differences in brain activation exist between the two conditions. Under the assumption that the differentially activated regions in the NS condition form a core pathway of conventional negative TAF, the regions of interest (ROIs) were defined at each cluster retrieved from the difference analysis map (NS > PS) and beta parameters at the peak of each cluster extracted. Next, Pearson's correlation analysis was performed to test for correlations between beta parameters and psychological measures. All statistical analyses were performed using the IBM SPSS Statistics for Windows, version 23 (IBM Corp., Armonk, NY, USA). Statistical sig- nificance was set at $p<0.05$.

\section{RESULTS}

\section{Psychological Assessment and Behavioral Data}

The baseline mean BDI and OCl-R scores were all within the normal range (Table 1) [24,29]. Participants showed no differences in emotional intensity of response $(3.8 \pm$ 0.3 vs. $3.8 \pm 0.3)$ and response time $(1.3 \pm 0.6$ vs. $1.4 \pm$ 0.6 seconds) between PS and NS conditions while performing the TAF task during the MRI scan.

\section{Common Brain Activation of Positive and Negative Statements Conditions}

Conjunction analysis using data from both the PS and NS conditions showed significant activation in the bilateral lingual and fusiform gyri, bilateral inferior frontal, and inferior orbitofrontal gyri (corresponding to the ventrolateral prefrontal cortex [vIPFC]), left middle frontal gyrus (corresponding to the dorsolateral PFC [dIPFC]), left supplementary motor area, left superior medial frontal gyrus (corresponding to dorsomedial PFC [dmPFC]), left mid-cingulate gyrus, bilateral angular and supramarginal gyri, left inferior parietal lobule, left middle temporal gy-

Table 1. Psychological assessments of healthy adults $(\mathrm{N}=31)$

\begin{tabular}{|c|c|c|}
\hline Psychological questionnaire & Mean & SD \\
\hline OC Inventory-Revised & 12.0 & 5.9 \\
\hline \multicolumn{3}{|l|}{ Dimensional OC Scale } \\
\hline Germs and contamination & 2.8 & 1.9 \\
\hline Responsible for harm or injury & 2.9 & 2.7 \\
\hline Unacceptable thoughts & 2.7 & 3.0 \\
\hline Symmetry, completeness & 1.2 & 2.6 \\
\hline Total & 10.4 & 8.2 \\
\hline Beck Depression Inventory & 4.3 & 4.2 \\
\hline \multicolumn{3}{|l|}{ Thought-Action Fusion Scale } \\
\hline Likelihood-self & 2.0 & 2.7 \\
\hline Likelihood-other & 2.5 & 3.5 \\
\hline Moral & 13.5 & 8.1 \\
\hline Total & 18.3 & 13.4 \\
\hline \multicolumn{3}{|l|}{ Behavioral data during MR experiment } \\
\hline \multicolumn{3}{|l|}{ Positive TAF condition } \\
\hline Response time, sec & 1.3 & 0.6 \\
\hline Intensity ${ }^{\mathrm{a}}$ & 3.8 & 0.3 \\
\hline \multicolumn{3}{|l|}{ Negative TAF condition } \\
\hline Response time, sec & 1.4 & 0.6 \\
\hline Intensity ${ }^{\mathrm{a}}$ & 3.8 & 0.3 \\
\hline
\end{tabular}

$\mathrm{SD}$, standard deviation; $\mathrm{OC}$, obsessive-compulsive; MR, magnetic resonance; TAF, thought-action fusion.

a Likert scores from 1 to 4. 
Table 2. Conjunction analysis of brain activation for both positive and negative TAF statements

\begin{tabular}{|c|c|c|c|c|c|c|}
\hline \multirow{2}{*}{ Region } & \multirow{2}{*}{ Left/Right } & \multirow{2}{*}{ Cluster size } & \multicolumn{3}{|c|}{ MNI coordinates $(\mathrm{mm})$} & \multirow{2}{*}{ Peak T } \\
\hline & & & $x$ & $y$ & z & \\
\hline \multirow{2}{*}{ Lingual gyrus } & L & 463 & -8 & -74 & -4 & 6.68 \\
\hline & $\mathrm{R}$ & 67 & 6 & -70 & 2 & 5.29 \\
\hline \multirow{2}{*}{ Fusiform gyrus } & L & 175 & -30 & -70 & -14 & 5.16 \\
\hline & $\mathrm{R}$ & 99 & 30 & -70 & -12 & 5.07 \\
\hline Supplementary motor area & L & 315 & -2 & 14 & 58 & 6.89 \\
\hline Superior medial frontal gyrus & L & 187 & -6 & 18 & 42 & 5.37 \\
\hline Middle frontal gyrus & L & 199 & -36 & 4 & 52 & 5.05 \\
\hline Inferior parietal lobe & L & 279 & -34 & -54 & 44 & 5.75 \\
\hline \multirow[t]{2}{*}{ Angular gyrus } & L & 112 & -58 & -54 & 32 & 5.77 \\
\hline & $\mathrm{R}$ & 38 & 38 & -60 & 48 & 4.73 \\
\hline \multirow[t]{2}{*}{ Supramarginal gyrus } & L & 219 & -56 & -54 & 30 & 5.27 \\
\hline & $\mathrm{R}$ & 48 & 58 & -48 & 28 & 4.69 \\
\hline Mid cingulate gyrus & L & 121 & -6 & 22 & 38 & 4.81 \\
\hline \multirow[t]{2}{*}{ Inferior frontal gyrus } & L & 237 & -48 & 20 & 0 & 5.55 \\
\hline & $\mathrm{R}$ & 21 & 42 & 20 & 6 & 4.83 \\
\hline \multirow[t]{2}{*}{ Inferior orbito-frontal gyrus } & L & 149 & -48 & 20 & -4 & 6.00 \\
\hline & $\mathrm{R}$ & 35 & 44 & 38 & -12 & 5.11 \\
\hline Middle temporal gyrus & L & 267 & -58 & -28 & -4 & 5.92 \\
\hline Thalamus & $\mathrm{L}$ & 28 & -8 & -10 & -2 & 5.02 \\
\hline Cerebellum posterior lobe & $\mathrm{R}$ & 110 & 10 & -74 & -24 & 6.07 \\
\hline
\end{tabular}

TAF, thought-action fusion; MNI, Montreal Neurological Institute.

$p<0.05$, false discovery rate (FDR) corrected for multiple comparison and minimum cluster size of 10 .

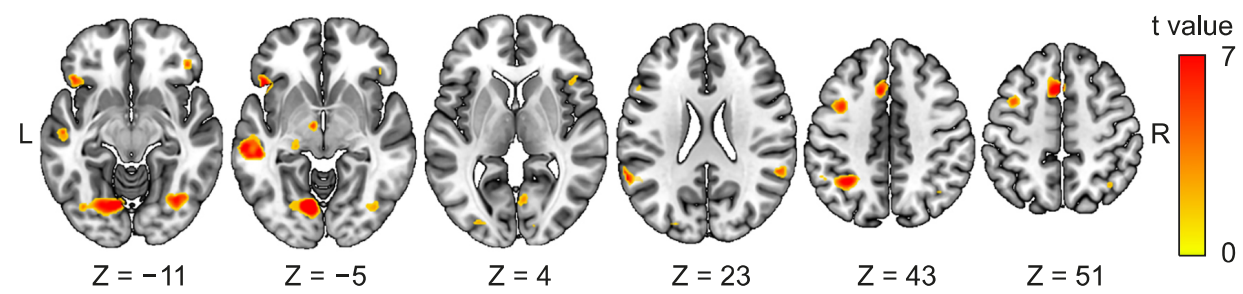

Fig. 1. Brain activation for conjunction analysis for conditions of both positive and negative statements. The map threshold was at a false discovery rate-corrected $p$ value $<0.05$ with a minimum cluster size of 10 .

rus, left thalamus, and cerebellum (Table 2 and Fig. 1).

\section{Differential Brain Activation between Positive and Negative Statements Conditions}

The NS > PS comparison revealed several new regions not shown in the conjunction analysis, including the bilateral precuneus and left rostral prefrontal gyrus, bilateral superior frontal gyri, right insula, caudate body, medial globus pallidus and thalamus, and left midbrain. It also revealed the same and adjacent regions shown in the conjunction analysis (i.e., several frontal subregions, superior and inferior parietal lobules, middle and superior temporal gyri, and the cerebellum). In contrast, the PS > NS comparison revealed no significant activation even at a lower threshold level of $p<0.005$ (uncorrected; Table 3 and Fig. 2).

\section{Relationship between Regional Activity and Psychological Measures}

ROls were used to investigate based on the differential analysis of this study whether activities in those regions were correlated with behavioral and psychological measures. The inferior orbitofrontal gyrus, insula, globus pallidus, and precuneus were correlated positively with response time and negatively with emotional intensity while subjects responded to the negative TAF task during the MRI scan (Fig. 3) in terms of behavioral data. However, all these regions revealed no relationships with either re- 
Table 3. Paired $t$ test of brain activation between positive and negative TAF statements

\begin{tabular}{|c|c|c|c|c|c|c|c|}
\hline \multirow{2}{*}{ Condition } & \multirow{2}{*}{ Region } & \multirow{2}{*}{ Left/Right } & \multirow{2}{*}{ Cluster size } & \multicolumn{3}{|c|}{ MNI coordinates (mm) } & \multirow{2}{*}{ Peak T } \\
\hline & & & & $x$ & $y$ & z & \\
\hline \multirow[t]{25}{*}{ Negative $>$ Positive } & Precuneus & $\mathrm{L}$ & 238 & -6 & -68 & 56 & 5.48 \\
\hline & & $\mathrm{R}$ & 29 & 10 & -64 & 21 & 4.20 \\
\hline & Middle frontal gyrus & $\mathrm{L}$ & 158 & -28 & 12 & 52 & 4.78 \\
\hline & Supplementary motor area & $\mathrm{L}$ & 40 & -12 & 4 & 52 & 5.00 \\
\hline & Superior parietal lobe & $\mathrm{L}$ & 76 & -22 & -72 & 50 & 5.05 \\
\hline & Superior frontal gyrus & $\mathrm{L}$ & 106 & -26 & 32 & 34 & 4.92 \\
\hline & & $\mathrm{R}$ & 29 & 22 & 42 & 26 & 4.91 \\
\hline & Rostral prefrontal gyrus & $\mathrm{L}$ & 31 & 0 & 60 & 6 & 4.94 \\
\hline & Mid cingulate gyrus & $\mathrm{L}$ & 36 & -4 & -42 & 44 & 5.01 \\
\hline & Inferior parietal lobe & $\mathrm{L}$ & 152 & -56 & -46 & 26 & 5.16 \\
\hline & Middle temporal gyrus & $\mathrm{L}$ & 128 & -60 & -32 & 4 & 5.55 \\
\hline & & $\mathrm{R}$ & 351 & 64 & -44 & -1 & 4.79 \\
\hline & Superior temporal gyrus & $\mathrm{L}$ & 72 & -58 & -28 & 4 & 5.25 \\
\hline & & $\mathrm{R}$ & 351 & 50 & -44 & 10 & 5.77 \\
\hline & Medial globus pallidus & $\mathrm{R}$ & 75 & 12 & 2 & -6 & 5.45 \\
\hline & Lingual gyrus & $\mathrm{L}$ & 48 & -16 & -52 & -6 & 4.80 \\
\hline & Inferior orbito-frontal cortex & $\mathrm{L}$ & 56 & -36 & 30 & -4 & 5.01 \\
\hline & Insula & $\mathrm{R}$ & 34 & 40 & 20 & -12 & 4.84 \\
\hline & Thalamus & $\mathrm{L}$ & 55 & -8 & -28 & 4 & 4.99 \\
\hline & & $\mathrm{R}$ & 32 & 6 & -24 & 4 & 4.75 \\
\hline & Caudate nucleus & $\mathrm{R}$ & 23 & 14 & 0 & 18 & 4.83 \\
\hline & Midbrain & $\mathrm{L}$ & 78 & -8 & -28 & -22 & 5.60 \\
\hline & Cerebellum anterior lobe & $\mathrm{R}$ & 91 & 22 & -46 & -26 & 5.24 \\
\hline & Cerebellum posterior lobe & $\mathrm{L}$ & 205 & -16 & -80 & -42 & 5.63 \\
\hline & & $\mathrm{R}$ & 147 & 28 & -72 & -42 & 5.27 \\
\hline Positive $>$ Negative & No significant activation & & & & & & \\
\hline
\end{tabular}

TAF, thought-action fusion; MNI, Montreal Neurological Institute.

$p<0.05$, false discovery rate (FDR) corrected for multiple comparison and minimum cluster size of 10 .

sponse time or emotional intensity in the positive TAF condition.

Precuneus activity was associated with the TAFS on baseline psychological measures (Fig. 2). The activity in the left inferior orbitofrontal gyrus $(r=0.50, p=0.004)$, right insula $(r=0.36, p=0.046)$, left superior $(r=0.45, p=$ $0.011)$ and middle $(r=0.39, p=0.03)$ frontal gyri, medial prefrontal cortex $(r=0.36, p=0.046)$, inferior parietal lobule $(r=0.39, p=0.03)$, and precuneus $(r=0.36, p=$ $0.047)$ were associated with total DOCS scores. The correlation between total DOCS scores and left inferior orbitofrontal gyrus was maintained after Bonferroni correction.

Additional exploratory correlation analyses were conducted to evaluate the relationships between each dimension of OCDs and activity in the brain regions (Table 4). The dimension of contamination was correlated with the superior frontal gyrus, inferior orbitofrontal gyrus, insula, globus pallidus, and precuneus; the dimension of responsibility with the inferior orbitofrontal gyrus and in- ferior parietal lobule; and the dimension of unacceptable thoughts with the superior and middle frontal gyri and rostral prefrontal gyrus. On the other hand, the dimension of symmetry showed no relationship with any brain regions.

\section{DISCUSSION}

This study had several main findings. First, both PS and NS showed shared activations in the fusiform and lingual gyri, midcingulate and superior medial frontal gyri, inferior orbitofrontal gyrus, and temporoparietal junction (TPJ). Second, the NS > PS comparison revealed additional activation in the precuneus and medial frontal pole, superior frontal gyrus, insula, globus pallidus, thalamus, and midbrain in terms of differential activations. On the other hand, the PS > NS comparison revealed no significant activation. Third, the activity in the precuneus correlated with the TAFS among the ROls activated in the NS > PS comparison. Together with those of the pre- 
A
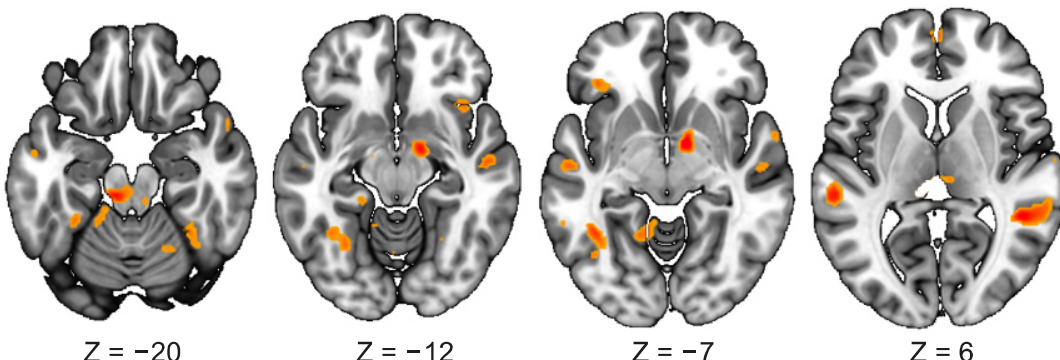

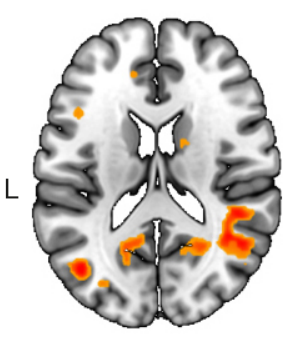

$Z=17$

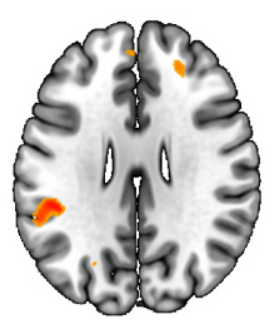

$Z=28$

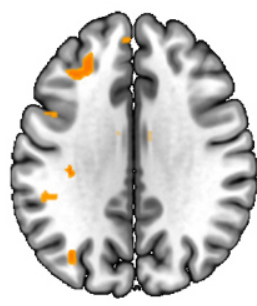

$Z=32$

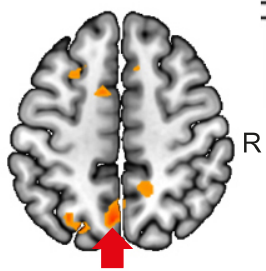

$Z=51$

B
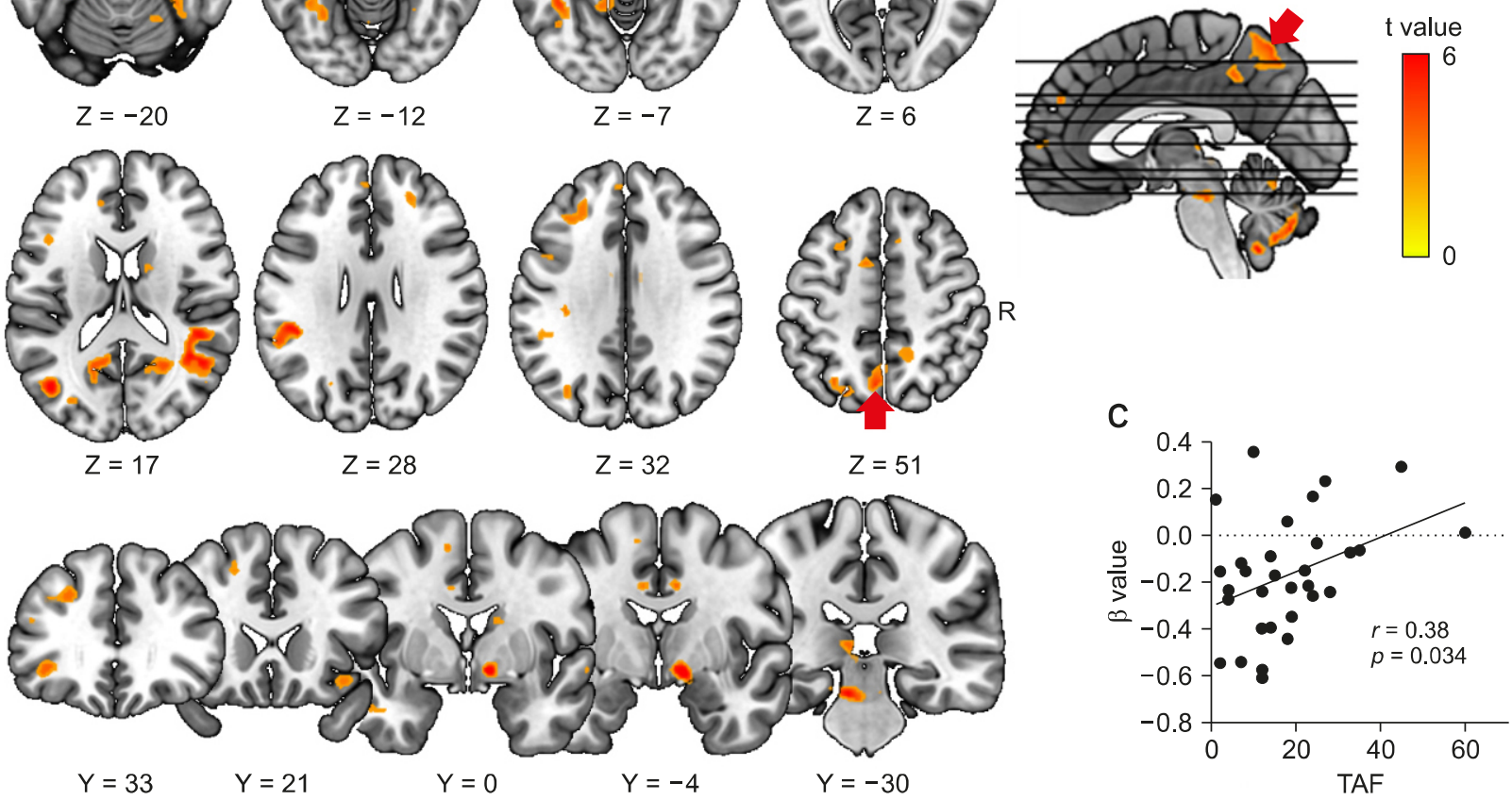

Fig. 2. Brain activation for the negative statements minus the positive statements condition. (A and B) show activations on the axial and coronal planes, respectively. Note that coronal images well-demonstrate the insula $(Y=21)$, caudate body, and globus pallidus $(Y=0)$, cingulate gyrus $(Y=-$ 4), and mediodorsal thalamus and midbrain $(Y=-30)$. (C) depicts the significant correlation between $\beta$ values in the precuneus (two red arrows in A) and scores of the Thought-Action Fusion Scale. The map threshold was at an uncorrected $p$ value $<0.005$ with a minimum cluster size of 10 . TAF, thought-action fusion.

cuneus, the activities in areas including the inferior orbitofrontal gyrus, several prefrontal regions, insula, and globus pallidus commonly showed significant relationships with the variables of the performance and OC symptom scores.

\section{Shared Activation of Positive and Negative TAF Statements}

Commonly activated regions in this study were the fusiform and lingual gyri (associated with the visual association), midcingulate and superior medial frontal gyri (associated with cognitive motor control, self-processing, and mentalizing), the inferior orbitofrontal gyrus (associated with cognitive reappraisal), and the inferior frontal cortex (associated with the mirror neuron system). The results of this study cannot suggest TAF-specific brain regions because most of these regions were overlapped with lan- guage and semantic networks [30-32]. However, the results of this study may contain several possible implications.

First, consistent with a previous study, this analysis showed activation of visual association areas such as the fusiform, lingual, and superior parietal lobes [20]. These structures have been linked to linguistic processing, the retrieval of visual imagery [33]. Also, these areas may have been more activated due to increased engagement of visual association areas in the negative TAF relative to that of the positive TAF condition. The results support the results of a previous study that suggests the importance of visual imagination in the TAF situation [20]. Second, specific roles related to TAF processing may exist although TPJ and vIPFC were crucial components for the language network. The left TPJ plays an important role in reasoning about other's beliefs, intentions, desires [34], and theory 


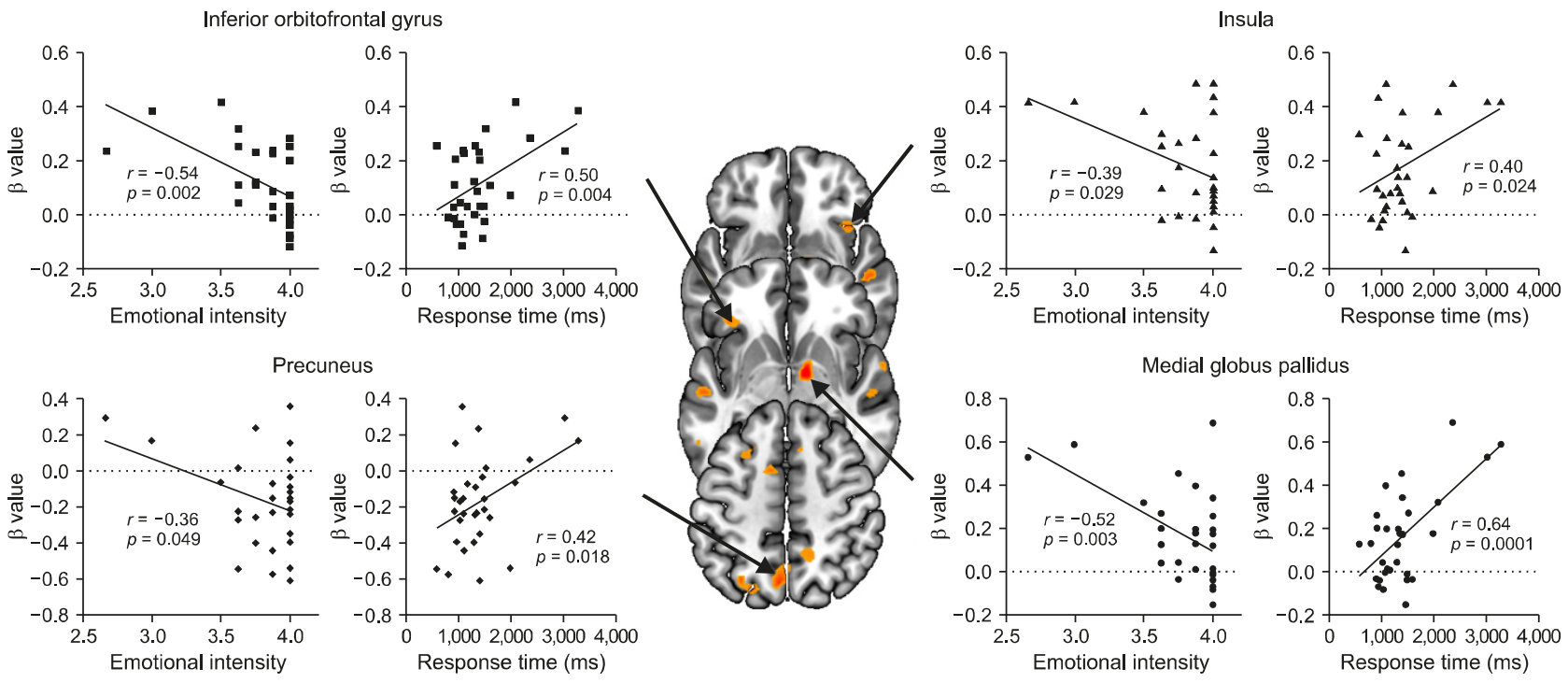

Fig. 3. Relationship between regional activity and the emotional intensity and response time during the magnetic resonance imaging scan in the condition of the negative statements minus the positive statements.

Table 4. Correlation between negative TAF-related activated regions and obsessive-compulsive dimensions

\begin{tabular}{lcccccccccc}
\hline \multicolumn{1}{c}{ OC symptom dimensions } & & Lt. SFG & Lt. MFG & MPFC & Lt. IOFG & Rt. Insula & Rt. mGP & Lt. IPL & Lt. Prec & Rt. Prec \\
\hline Germs and contamination & $r$ & 0.462 & 0.239 & 0.307 & 0.541 & 0.428 & 0.393 & 0.195 & 0.008 & 0.392 \\
& $p$ & 0.009 & 0.195 & 0.093 & 0.002 & 0.016 & 0.029 & 0.294 & 0.966 & 0.029 \\
Responsibility for harm, injury & $r$ & 0.320 & 0.298 & 0.176 & 0.472 & 0.267 & 0.296 & 0.416 & 0.088 & 0.287 \\
& $p$ & 0.079 & 0.103 & 0.344 & 0.007 & 0.147 & 0.106 & 0.020 & 0.639 & 0.117 \\
Unacceptable thoughts & $r$ & 0.386 & 0.373 & 0.381 & 0.308 & 0.256 & 0.148 & 0.352 & -0.032 & 0.191 \\
& $p$ & 0.032 & 0.039 & 0.034 & 0.092 & 0.165 & 0.426 & 0.052 & 0.864 & 0.304 \\
Symmetry, completeness & $r$ & 0.306 & 0.316 & 0.297 & 0.349 & 0.261 & 0.234 & 0.254 & -0.173 & 0.336 \\
& $p$ & 0.094 & 0.084 & 0.104 & 0.054 & 0.156 & 0.205 & 0.167 & 0.353 & 0.065 \\
\hline
\end{tabular}

TAF, thought-action fusion; OC, obsessive-compulsive; Lt., left; Rt., right; SFG, superior frontal gyrus; MFG, middle frontal gyrus; IOFG, inferior orbitofrontal gyrus; mGP, medial globus pallidus; IPL, inferior parietal lobule; Prec, precuneus.

of mind [35]. Also, vIPFC has been implicated as a key node of the regulatory cognitive control networks, particularly the selection and inhibition of appraisals, during cognitive reappraisal of emotion [36]. Therefore, these regions may be related to feeling the pain of others and monitoring the appropriateness of the response aroused by the TAF stimuli.

\section{Differential Activation in Positive and Negative TAF Conditions}

Interestingly, no significant activation was noted in the positive TAF $>$ negative TAF contrast. In an fMRI study assessing empathic response to others experiencing pain and happiness depicted in pictures, empathy for pain activated much broader areas than empathy for happiness, although positive empathy selectively activated regions as- sociated with positive affect such as the ventromedial PFC [37]. A previous EEG study revealed that although the degree of empathy elicited by a negative event involving either a stranger or close friend was larger than that of the control, the empathic response elicited by the positive event involving a stranger was not larger than that of the control [38]. This and previous studies suggest that individuals show greater neural response to negative than to positive events.

In addition to greater neural activation in general, the negative TAF condition revealed differentially activated brain regions. First, the precuneus was the area that the negative TAF was more robustly recruited than the positive TAF. The TAF paradigm of this study showed that the precuneus is thought to play an important role in vivid autobiographical reminiscence [39]. Also, the precuneus 
plays a role in mental imagery that has been suggested to extend to the imagery modeling other people's views [40]. The third-person perspective recruited more activity of the precuneus compared to the first-person perspective [41]. The precuneus, together with the superior frontal gyrus and orbitofrontal cortex (OFC), is activated when people make judgments that require understanding whether to act out of empathy and forgiveness [42]. Thus, the negative TAF may preferentially recruit the precuneus for sensing or judging others' pain in a negative situation. Accordingly, the activity in the left precuneus (corresponding to Brodmann area 7) was correlated with the TAFS in this study.

Second, this contrast demonstrated lateral orbitofrontal-striatal loops, such as that involving the lateral OFC, the body of caudate, medial globus pallidus, and mediodorsal nucleus of the thalamus [43,44]. Notably, these regions were not evident in the conjunction analysis although the lateral OFCs were bilaterally activated. Considering the TAF task of this study, these results were consistent with an fMRI study demonstrating that activation of the lateral OFC was tightly associated with reappraisal success when participants were forced to cognitively reappraise an aversive image [45]. Therefore, the roles of lateral OFC may monitor the appropriateness of responses aroused by the TAF situation and control the transition between goal-directed and habitual behaviors following the revaluation of various outcomes within the context of decision making.

Third, the midbrain tegmentum and anterior insula were differentially activated in the negative TAF condition in this study [46]. Interoception and emotional feelings may share a common route through the brainstem to the anterior insular cortex $[47,48]$. These regions are components of salience network (SN) together with dIPFC. Working together, the subcortical components of the SN communicate extensively with their cortical counterparts to select salient (motivationally relevant) stimuli and to enable such stimuli to direct cognition and behavior. This architecture enables the $\mathrm{SN}$ to play its central roles in salience detection, cognitive control, and the selection and/or inhibition of behavioral responses during healthy brain function. These findings suggest that salience detection can be a crucial component for TAF processing when characterizing the TAF stimuli of this study as one that has negative emotional valence with high ambiguity.

Differential points should be noticed although the re- sults of this study seem to correspond to negative emotional processing. The limbic regions, especially the amygdala, were commonly activated by negative emotional stimuli $[49,50]$. A recent meta-analysis showed that negative emotion recruited more activity in the amygdala, insula, and mPFC compared with positive emotion [51]. However, previous [20] and current studies showed that the TAF stimuli consistently induced activity in the precuneus, lateral prefrontal structures, and basal ganglia including the caudate nucleus. These components can be related to perspective-taking [41], empathy [42], and obsessional thought regarding the cortico-striato-thalamocortical circuit rather than the classical emotional network.

\section{Regions Associated with the Performance of Negative TAF Tasks}

Activities in the regions of the globus pallidus, inferior orbitofrontal gyrus, insula, and precuneus commonly showed positive relationships with response time and negative correlations with emotional intensity when performing a negative TAF task. In other words, these four areas were used more actively when participants took a longer time and evaluated negative TAF statements less negatively. It is speculated that these four regions are pivotal areas for determining the speed of response from evaluating stimuli (i.e., negative TAF statements) to taking actions (i.e., pushing a button).

\section{Relationships between Activated Areas and Obsessive-compulsive Symptoms}

Although the participants of this study were healthy subjects, their responses have value for addressing the relationships between TAF-related activation and OC symptoms because the concept of TAF was originally developed to understand cognitive reappraisal style in OCD. In addition to the four aforementioned regions, the superior and middle frontal gyri (dIPFC), medial PFC, and inferior parietal lobule were correlated with DOCS scores. A recent meta-analysis showed that all these regions were implicated in functional imaging studies suggesting that OCD-related brain dysfunction involves increased affective and self-referential processing, enhanced salience and habitual responding, and blunted cognitive control [52]. According to this meta-analysis, positive and negative TAF paradigm includes both non-affective and affective aspects of the cognitive task. Moreover, though pre- 
liminary in nature, each OC dimension has its correlation pattern with the ROls. Notably, the contamination dimension correlated with the insula (associated with disgust), the responsibility dimension with the inferior parietal lobule (associated with perspective taking), and the unacceptable thought dimension with the dIPFC and dmPFC (associated with cognitive control).

This study has several limitations. First, the results of conjunctional analyses should be cautiously interpreted. The activity of the brain regions related to the language network can be included in the results of this study because an appropriate contrast, such as sentence stimuli, was not used in our conjunctional analysis. Second, habituation effects could have weakened the positive TAF-related brain response. The positive TAF statements were implemented after the negative TAF statements. Even though habituation effects may exist, this study firstly confirmed a shared brain network related to the TAF response in both positive and negative aspects. Third, the level of belief of the subjects on statements believed to likely happen can be a crucial component of the TAF paradigm of this study. However, the participants were not asked directly. Thus, the relationship between brain activity and the subjects' strength of false beliefs induced by the TAF task could not be explained. Fourth, the generalizability of the results could be limited by the sample characteristics of the all-male subjects in this study. However, previous literature has suggested that TAF belief may not be dependent on sex [53,54]. Future studies are needed to compensate for the limitations of the present study and confirm its findings for a better understanding of the biological mechanism underlying the TAF responses connected to various psychopathologies.

In conclusion, negative TAF induced more brain activation compared to positive TAF. Relative to positive TAF, negative TAF recruit additional regions for self-referential processing, salience, and habitual responding, which may contribute to the activation of the belief that a negative thought increases the probability of that negative outcome. In addition, among these regions, the activity of precuneus that was involved in mental imagery, perspective taking, and emphatic response was directly associated with the TAF score.

\section{Acknowledgments}

This work was supported by the National Research
Foundation of Korea (NRF) grant funded by the Korean government (MSIP) (NRF-2018R1A2B6007374).

\section{Conflicts of Interest}

No potential conflict of interest relevant to this article was reported.

\section{Author Contributions}

Conceptualization: Sang Won Lee, Yongmin Chang, and Seung Jae Lee. Data acquisition: Sang Won Lee, Hyunsil Cha, Tae Yang Jang, Eunji Kim, and Seung Jae Lee. Formal analysis: Hyunsil Cha, Eunji Kim, Huijin Song, and Yongmin Chang. Writing the original draft: Sang Won Lee, Hyunsil Cha, and Seung Jae Lee. Writing, reviewing, and editing: Sang Won Lee, Hyunsil Cha, Yongmin Chang and Seung Jae Lee.

\section{ORCID}

Sang Won Lee

Hyunsil Cha

Tae Yang Jang

Eunji Kim

Huijin Song

Yongmin Chang

Seung Jae Lee

https://orcid.org/0000-0002-3537-7110 https://orcid.org/0000-0002-6887-8595 https://orcid.org/0000-0001-9106-9414 https://orcid.org/0000-0002-2677-4325 https://orcid.org/0000-0001-7167-115X https://orcid.org/0000-0002-0585-8714 https://orcid.org/0000-0003-3648-9824

\section{REFERENCES}

1. Herzberg KN, Sheppard SC, Forsyth JP, Credé M, Earleywine $\mathrm{M}$, Eifert $\mathrm{GH}$. The Believability of Anxious Feelings and Thoughts Questionnaire (BAFT): a psychometric evaluation of cognitive fusion in a nonclinical and highly anxious community sample. Psychol Assess 2012;24:877-891.

2. Reuman L, Buchholz J, Abramowitz JS. Obsessive beliefs, experiential avoidance, and cognitive fusion as predictors of obsessive-compulsive disorder symptom dimensions. J Context Behav Sci 2018;9:15-20.

3. Reuman L, Buchholz J, Blakey S, Abramowitz JS. Uncertain and fused: cognitive fusion, thought-action fusion, and the intolerance of uncertainty as predictors of obsessive-compulsive symptom dimensions. J Cogn Psychother 2017;31:191-203.

4. Eifert GH, Forsyth JP. Acceptance and commitment therapy for anxiety disorders: a practitioner's treatment guide to using mindfulness, acceptance, and values-based behavior change strategies. Oakland:New Harbinger Publications;2005.

5. Shafran R, Thordarson DS, Rachman S. Thought-action fusion in obsessive compulsive disorder. J Anxiety Disord 1996;10: 379-391.

6. Craig JC, Lafreniere KD. Positive thought-action fusion as an independent construct. Personal Individ Differ 2016;94:228- 
236.

7. Shafran R, Rachman S. Thought-action fusion: a review. J Behav Ther Exp Psychiatry 2004;35:87-107.

8. Berle D, Starcevic V. Thought-action fusion: review of the literature and future directions. Clin Psychol Rev 2005;25:263284.

9. Bailey BE, Wu KD, Valentiner DP, McGrath PB. Thought-action fusion: structure and specificity to OCD. J ObsessCompuls Relat Disord 2014;3:39-45.

10. Salkovskis PM. Obsessional-compulsive problems: a cognitive-behavioural analysis. Behav Res Ther 1985;23:571-583.

11. Clark DA, Purdon CL. The assessment of unwanted intrusive thoughts: a review and critique of the literature. Behav Res Ther 1995;33:967-976.

12. Rachman S, Shafran R. Cognitive distortions: thought-action fusion. Clin Psychol Psychother 1999; 6:80-85.

13. Hazlett-Stevens H, Zucker BG, Craske MG. The relationship of thought-action fusion to pathologicial worry and generalized anxiety disorder. Behav Res Ther 2002;40:1199-1204.

14. Lee HJ, Cougle JR, Telch MJ. Thought-action fusion and its relationship to schizotypy and OCD symptoms. Behav Res Ther 2005:43:29-41.

15. Abramowitz JS, Whiteside S, Lynam D, Kalsy S. Is thought-action fusion specific to obsessive-compulsive disorder?: a mediating role of negative affect. Behav Res Ther 2003;41:10691079.

16. Ghamari Kivi H, Mohammadipour Rik N, Sadeghi Movahhed F. Explanation of obsessive-compulsive disorder and major depressive disorder on the basis of thought-action fusion. Iran J Psychiatry Behav Sci 2013;7:44-50.

17. Amir N, Freshman M, Ramsey B, Neary E, Brigidi B. Thoughtaction fusion in individuals with OCD symptoms. Behav Res Ther 2001;39:765-776.

18. Sanavio E. Obsessions and compulsions: the Padua Inventory. Behav Res Ther 1988;26:169-177.

19. Jones R, Bhattacharya J. A role for the precuneus in thoughtaction fusion: evidence from participants with significant obsessive-compulsive symptoms. Neuroimage Clin 2013;4: 112-121.

20. Lee SW, Cha H, Chung Y, Kim E, Song H, Chang Y, et al. The neural correlates of thought-action fusion in healthy adults: a functional magnetic resonance imaging study. Depress Anxiety 2019;36:732-743.

21. Rachman S, Shafran R, Mitchell D, Trant J, Teachman B. How to remain neutral: an experimental analysis of neutralization. Behav Res Ther 1996;34:889-898.

22. Lee S. The relationships of OC symptoms with moral and causal responsibility and with omission. Seoul:Seoul National University;2000. [Thesis].

23. Foa EB, Huppert JD, Leiberg S, Langner R, Kichic R, Hajcak G, et al. The Obsessive-Compulsive Inventory: development and validation of a short version. Psychol Assess 2002;14:485496.
24. Woo CW, Kwon SM, Lim YJ, Shin MS. The ObsessiveCompulsive Inventory-Revised (OCl-R): psychometric properties of the Korean version and the order, gender, and cultural effects. J Behav Ther Exp Psychiatry 2010;41:220-227.

25. Abramowitz JS, Deacon BJ, Olatunji BO, Wheaton MG, Berman NC, Losardo D, et al. Assessment of obsessive-compulsive symptom dimensions: development and evaluation of the Dimensional Obsessive-Compulsive Scale. Psychol Assess 2010;22:180-198.

26. Kim HW, Kang JI, Kim SJ, Jhung K, Kim EJ, Kim SJ. A validation study of the Korean-version of the Dimensional ObsessiveCompulsive Scale. J Korean Neuropsychiatr Assoc 2013;52: 130-142.

27. Beck AT, Ward CH, Mendelson M, Mock J, Erbaugh J. An inventory for measuring depression. Arch Gen Psychiatry 1961; 4:561-571.

28. Lee $\mathrm{YH}$, Song JY. A study of the reliability and the validity of the BDI, SDS, and MMPI-D scales. Korean I Clin Psychol 1991;10:98-113.

29. Rhee MK, Lee YH, Jung HY, Choi JH, Kim SH, Kim YG, et al. A standardization study of Beck depression inventory - Korean version (K-BDI): validity. Korean / Psychopathol 1995; 4:96-104.

30. Branco P, Seixas D, Castro SL. Mapping language with resting-state functional magnetic resonance imaging: a study on the functional profile of the language network. Hum Brain Mapp 2020;41:545-560.

31. Hagoort $\mathrm{P}$, Hald L, Bastiaansen M, Petersson KM. Integration of word meaning and world knowledge in language comprehension. Science 2004;304:438-441.

32. Lee SW, Jeong B, Park JI, Chung GH, Lee HJ, Cui Y, et al. Alteration of semantic networks during swear words processing in schizophrenia. Clin Psychopharmacol Neurosci 2019; 17:64-73.

33. Leshikar ED, Duarte A, Hertzog C. Task-selective memory effects for successfully implemented encoding strategies. PLoS One 2012; 7:e38160.

34. Gallagher HL, Happé F, Brunswick N, Fletcher PC, Frith U, Frith CD. Reading the mind in cartoons and stories: an FMRI study of 'theory of mind' in verbal and nonverbal tasks. Neuropsychologia 2000;38:11-21.

35. Saxe R, Kanwisher N. People thinking about thinking people. The role of the temporo-parietal junction in "theory of mind". Neuroimage 2003;19:1835-1842.

36. Buhle JT, Silvers JA, Wager TD, Lopez R, Onyemekwu C, Kober $\mathrm{H}$, et al. Cognitive reappraisal of emotion: a meta-analysis of human neuroimaging studies. Cereb Cortex 2014;24: 2981-2990.

37. Motomura Y, Takeshita A, Egashira Y, Nishimura T, Kim YK, Watanuki S. Interaction between valence of empathy and familiarity: is it difficult to empathize with the positive events of a stranger? J Physiol Anthropol 2015;34:13.

38. Morelli SA, Rameson LT, Lieberman MD. The neural compo- 
nents of empathy: predicting daily prosocial behavior. Soc Cogn Affect Neurosci 2014;9:39-47.

39. Sreekumar V, Nielson DM, Smith TA, Dennis SJ, Sederberg $\mathrm{PB}$. The experience of vivid autobiographical reminiscence is supported by subjective content representations in the precuneus. Sci Rep 2018;8:14899.

40. Vogeley K, May M, Ritzl A, Falkai P, Zilles K, Fink GR. Neural correlates of first-person perspective as one constituent of human self-consciousness. J Cogn Neurosci 2004; 16:817-827.

41. Ruby P, Decety J. Effect of subjective perspective taking during simulation of action: a PET investigation of agency. Nat Neurosci 2001; 4:546-550.

42. Farrow TF, Zheng Y, Wilkinson ID, Spence SA, Deakin JF, Tarrier $\mathrm{N}$, et al. Investigating the functional anatomy of empathy and forgiveness. Neuroreport 2001;12:2433-2438.

43. Fettes P, Schulze L, Downar J. Cortico-striatal-thalamic loop circuits of the orbitofrontal cortex: promising therapeutic targets in psychiatric illness. Front Syst Neurosci 2017;11:25.

44. Menzies L, Chamberlain SR, Laird AR, Thelen SM, Sahakian $\mathrm{BJ}$, Bullmore ET. Integrating evidence from neuroimaging and neuropsychological studies of obsessive-compulsive disorder: the orbitofronto-striatal model revisited. Neurosci Biobehav Rev 2008;32:525-549.

45. Wager TD, Davidson ML, Hughes BL, Lindquist MA, Ochsner $\mathrm{KN}$. Prefrontal-subcortical pathways mediating successful emotion regulation. Neuron 2008; 59:1037-1050.

46. Parvizi J, Damasio A. Consciousness and the brainstem.
Cognition 2001;79:135-160.

47. Craig AD. Interoception: the sense of the physiological condition of the body. Curr Opin Neurobiol 2003;13:500-505.

48. Drake MJ, Fowler CJ, Griffiths D, Mayer E, Paton JF, Birder L. Neural control of the lower urinary and gastrointestinal tracts: supraspinal CNS mechanisms. Neurourol Urodyn 2010;29: 119-127.

49. Preckel K, Trautwein FM, Paulus FM, Kirsch P, Krach S, Singer $\mathrm{T}$, et al. Neural mechanisms of affective matching across faces and scenes. Sci Rep 2019;9:1492.

50. Yang TT, Menon V, Eliez S, Blasey C, White CD, Reid AJ, et al. Amygdalar activation associated with positive and negative facial expressions. Neuroreport 2002;13:1737-1741.

51. Lindquist KA, Satpute AB, Wager TD, Weber J, Barrett LF. The brain basis of positive and negative affect: evidence from a meta-analysis of the human neuroimaging literature. Cereb Cortex 2016;26:1910-1922.

52. Rasgon A, Lee WH, Leibu E, Laird A, Glahn D, Goodman W, et al. Neural correlates of affective and non-affective cognition in obsessive compulsive disorder: a meta-analysis of functional imaging studies. Eur Psychiatry 2017;46:25-32.

53. Muris P, Meesters C, Rassin E, Merckelbach H, Campbell J. Thought-action fusion and anxiety disorders symptoms in normal adolescents. Behav Res Ther 2001;39:843-852.

54. Yorulmaz O, Yilmaz AE, Gençöz T. Psychometric properties of the Thought-Action Fusion Scale in a Turkish sample. Behav Res Ther 2004;42:1203-1214. 